\title{
A New Model for Calculating Well Trajectory
}

\author{
Samir M. Khaled, PhD \\ Mining and Petroleum Engineering Department, Faculty of Engineering, Al-Azhar University
}

\begin{abstract}
There are many calculation techniques for determining the trajectory of the wellbore. The most common techniques are tangential method, balanced method, angle averaging method, radius of curvature method and minimum curvature method. This paper presents a comparison study among five different models used to calculate the well trajectory using actual field data for different well profiles (build \& hold, S shape and modified S trajectory). A new model was developed for calculating the well trajectory and compared with different models mentioned above using the same data and the same well profiles. The results obtained during the present work indicated that the new model is more accurate than the other methods for calculating the true vertical depth. At calculate Horizontal displacement, northing and easting different methods give very close agreement, with the exception of the tangential method.
\end{abstract}

Keywords: Trajectory, tangential method, balanced method, angle averaging method.

\section{INTRODUCTION}

Controlled directional drilling can be defined as a science of deviating a well bore along a planned course to a subsurface target whose location is a given lateral distance and direction from the vertical. At a specified vertical depth, this definition is the fundamental concept of held as close to vertical as possible as well as a deliberately planned deviation from the vertical.

To control the deviating a well bore, a number of survey calculation methods have been used in directional drilling. Most common type: Tangential, Average angle, Balanced, Radius of Curvature and Minimum Curvature method.

Regardless of which conventional survey method is used, the following three pieces of information are known at the end of a successful survey, Measured Depth, Borehole Inclination and Borehole Azimuth (corrected to relevant North). In order to ascertain the latest bottom-hole position, it is necessary to perform a survey calculation which includes the three inputs listed above. Only then can the latest bottom-hole coordinates be plotted on the directional well plot (True vertical depth vs Horizontal displacement on the vertical plot and (Northing N/S vs Easting E/W) rectangular coordinates on horizontal plot).

The more common methods that have been adopted are described in the following paragraphs.

Tangential method: The mathematics uses the inclination and direction at a survey station and assumes the inclination angle remain constant over the measured depth.

Average angle method: When using the average angle method, the inclination and azimuth at the lower and upper survey stations are mathematically averaged, and then the wellbore course is assumed to be tangential to the average inclination and azimuth. The calculations are very similar to the tangential method

Balanced tangential method: This model uses the survey data from both the upper and lower stations. The model assumes that the well path lies along two equal length, straight line segments. The inclination and direction of each segment is given by the corresponding survey station. The tangential model is therefore applied twice - once to the upper half, once to the lower half. $[2,9]$

Radius of curvature method: The method assumes the wellbore course is a smooth curve between the upper and lower survey stations. The curvature of the arc is determined by the survey inclinations and azimuths at the upper and lower survey stations. The length of the arc between two stations is the measured depth between surveys. $[1,2,5,7,9]$

The equations are complicated and are not easily calculated in the field without a programmable calculator or computer. In the equations, the inclination and azimuth are entered as degrees. $[1,2,5,7,9]$

Minimum curvature method: The minimum curvature method is similar to the radius of curvature method in that it assumes that the wellbore is a curved path between the two survey points. The minimum curvature method uses the same equations as the balanced tangential multiplied by a ratio factor which is defined by the curvature of the wellbore. Like the radius of curvature, the equations are more complicated and not easily calculated in the field without the aid of a programmable calculator or computer. $[1,2,5,7,9]$

The main objective of the present work is to introduce a new developed model based on both average angles and balanced tangential methods. In addition, the suggested model is to maximize the advantages of the previous models by combining them into the new model. Through the new model called as balanced tangential \& average angle model, The new developed model assume that the actual well path can be approximated by two straight line segments of equal length and inclination angles at upper and lower segment have been averaged, also azimuth angles have been averaged at upper and lower segment. 


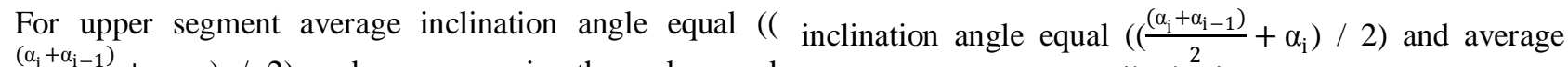
$\left.\left.\frac{\left(\alpha_{i}+\alpha_{i-1}\right)}{2}+\alpha_{i-1}\right) / 2\right)$ and average azimuth angle equal azimuth angle equal $\left(\left(\frac{\left(A_{i}+A_{i-1}^{2}\right)}{2}+A_{i}\right) / 2\right)$. As shown in $\left(\left(\frac{\left(A_{i}+A_{i-1}\right)}{2}+A_{i-1}\right) / 2\right)$. For lower segment average figure 1

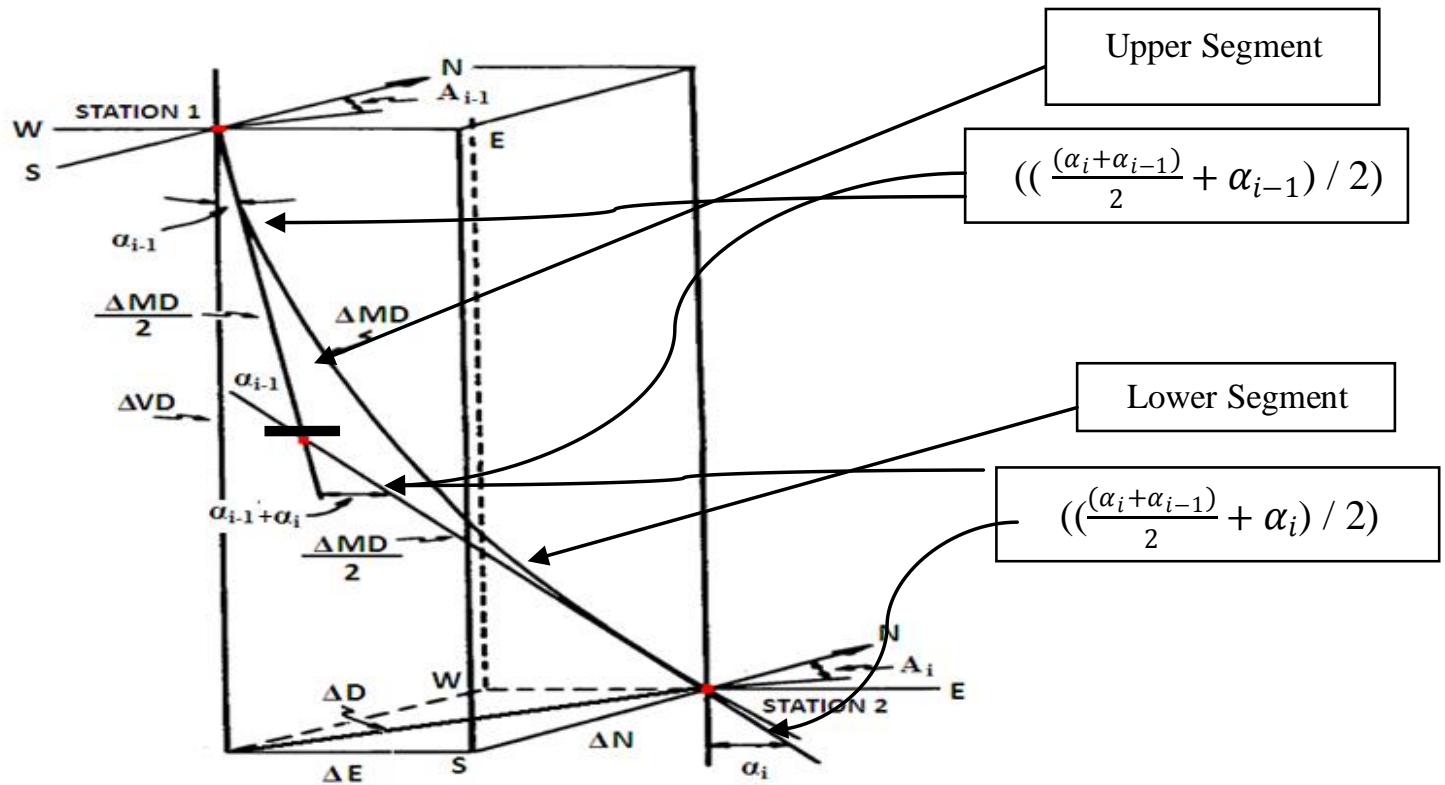

Figure 1 New model (balanced tangential \& average angle model)

From plotting in Fig. 1 the equations for new model can be summarized as follows: Vertical distance between any two points on wellpath:

$$
\Delta \mathrm{VD}_{\mathrm{i}}=\frac{\Delta \mathrm{MD}_{\mathrm{i}}}{2}\left(\cos \left(\left(\frac{\left(\alpha_{\mathrm{i}}+\alpha_{\mathrm{i}-1}\right)}{2}+\alpha_{\mathrm{i}-1}\right) / 2\right)+\cos \left(\left(\frac{\left(\alpha_{\mathrm{i}}+\alpha_{\mathrm{i}-1}\right)}{2}+\alpha_{\mathrm{i}}\right) / 2\right)\right)
$$

Total true vertical depth: $\operatorname{TVD}=\sum_{\mathrm{i}=1}^{\mathrm{n}} \Delta \mathrm{VDi}$

Horizontal distance between any two points on wellpath:

$$
\left.\left.\Delta \mathrm{D}_{\mathrm{i}}=\frac{\Delta \mathrm{MD}_{\mathrm{i}}}{2}\left(\sin \left(\frac{\left(\alpha_{\mathrm{i}}+\alpha_{\mathrm{i}-1}\right)}{2}+\alpha_{\mathrm{i}-1}\right) / 2\right)+\sin \left(\frac{\left(\alpha_{\mathrm{i}}+\alpha_{\mathrm{i}-1}\right)}{2}+\alpha_{\mathrm{i}}\right) / 2\right)\right)
$$

Total Horizontal distance: $\quad H D=\sum_{\mathrm{i}=1}^{\mathrm{n}} \Delta \mathrm{Di}$

East direction between any two points on wellpath:

$$
\begin{aligned}
E_{i}=\frac{\Delta M D_{i}}{2}((\sin ( & \left.\left.\left(\frac{\left(\alpha_{i}+\alpha_{i-1}\right)}{2}+\alpha_{i-1}\right) / 2\right) * \sin \left(\left(\frac{\left.A_{i}+A_{i-1}\right)}{2}+A_{i-1}\right) / 2\right)\right) \\
& \left.\left.+\left(\sin \left(\frac{\left(\alpha_{i}+\alpha_{i-1}\right)}{2}+\alpha_{i}\right) / 2\right) * \sin \left(\left(\frac{\left(A_{i}+A_{i-1}\right)}{2}+A_{i}\right) / 2\right)\right)\right)
\end{aligned}
$$

Total East direction: $\quad \mathrm{E}=\sum_{\mathrm{i}=1}^{\mathrm{n}} \Delta \mathrm{Ei} \quad$ North direction between any two points on wellpath:

$$
\begin{aligned}
\Delta \mathrm{N}_{\mathrm{i}}=\frac{\Delta \mathrm{MD}_{\mathrm{i}}}{2}(( & \left.\sin \left(\left(\frac{\left(\alpha_{\mathrm{i}}+\alpha_{\mathrm{i}-1}\right)}{2}+\alpha_{\mathrm{i}-1}\right) / 2\right) * \cos \left(\left(\frac{\left(\mathrm{A}_{\mathrm{i}}+\mathrm{A}_{\mathrm{i}-1}\right)}{2}+\mathrm{A}_{\mathrm{i}-1}\right) / 2\right)\right) \\
& \left.+\left(\sin \left(\left(\frac{\left(\alpha_{\mathrm{i}}+\alpha_{\mathrm{i}-1}\right)}{2}+\alpha_{\mathrm{i}} / 2\right)\right) * \cos \left(\left(\frac{\left(\mathrm{A}_{\mathrm{i}}+\mathrm{A}_{\mathrm{i}-1}\right)}{2}+\mathrm{A}_{\mathrm{i}}\right) / 2\right)\right)\right)
\end{aligned}
$$

Total North direction: $\quad \mathrm{N}=\sum_{\mathrm{i}=1}^{\mathrm{n}} \Delta \mathrm{Ni}$ 


\section{International Advanced Research Journal in Science, Engineering and Technology}

ISO 3297:2007 Certified

Vol. 3, Issue 11, November 2016

Available data

In this study, we will introduce the basic data about the three fields from which the wells were taken as one well for every field, as showing in the table 1

Table (1) General information about wells which are used in this study

\begin{tabular}{|l|l|l|l|}
\hline Well No & A 92 & FD 31 & 5 I17 \\
\hline Field & 1 & 2 & 3 \\
\hline Well profile & Type I(Build and Hold) & Type II (Build, Hold and Drop) & Type III (Deep kick off and Build) \\
\hline & & & \\
\hline
\end{tabular}

\section{CONCLUSIONS}

Compares five the different methods with new model The results obtained during the present work indicated using data taken from actual fields (Measured Depth, that:

Borehole Inclination and Borehole Azimuth (corrected to 1. New model is more accurate method to calculate true relevant North). at different type of well profile build \& vertical depth (TVD) for all type of wells trajectory

hold, S shape and Deep Kick off and Build. The results of 2. The differences among new model, average angle, various calculation method tabulated in tables from 1-3. Results calculated by minimum curvature method used as reference to comparison. balanced, and radius of curvature, methods are so small, that any of the methods could be used for calculating the trajectory.

3. The least accurate method is tangential method for all types of trajectory.

Table 2 Comparison of Accuracy of Various Calculation Method with New Model for Build and hold well (A 92)

\begin{tabular}{|c|c|c|c|c|c|c|c|c|}
\hline \multirow{2}{*}{$\begin{array}{l}\text { Method } \\
\text { Minimum Curvature }\end{array}$} & \multicolumn{2}{|c|}{$\begin{array}{l}\text { TVD \& difference } \\
\text { from } \mathrm{MC} *\end{array}$} & \multicolumn{2}{|c|}{$\begin{array}{l}\text { HD \& difference } \\
\text { from } \mathrm{MC} *\end{array}$} & \multicolumn{2}{|c|}{$\begin{array}{l}\text { North } \\
\text { displacement } \\
\text { \& difference from } \\
\text { MC* }\end{array}$} & \multicolumn{2}{|c|}{$\begin{array}{l}\text { East } \\
\text { displacement \& } \\
\text { difference from } \\
\text { MC* }\end{array}$} \\
\hline & 14147.07 & 0.00 & 3160.05 & 0.00 & 3126.98 & 0.00 & -455.98 & 0.00 \\
\hline Tangential & 14139.44 & 7.63 & 3241.06 & -81.01 & 3152.49 & -25.51 & -459.82 & 3.84 \\
\hline Average Angle & 14147.16 & -0.09 & 3215.14 & -55.09 & 3102.41 & 24.57 & -454.25 & -1.73 \\
\hline Balance & 14146.94 & 0.13 & 3215.08 & -55.03 & 3116.90 & 10.08 & -455.91 & -0.07 \\
\hline New Model & 14147.10 & -0.03 & 3215.12 & -55.07 & 3114.98 & 12.00 & -455.78 & -0.20 \\
\hline Radius of Curvature & 14147.04 & 0.03 & 3215.19 & -55.14 & 3114.80 & 12.18 & -455.97 & -0.01 \\
\hline
\end{tabular}

* Minimum Curvature

Table 3 Comparison of Accuracy of Various Calculation Method with New Model for Build hold and drop well (FD 31)

\begin{tabular}{|l|l|l|l|l|l|l|l|l|}
\hline Method & $\begin{array}{l}\text { TVD } \\
\text { difference from } \\
\text { MC* }\end{array}$ & $\begin{array}{l}\text { HD \& difference } \\
\text { from MC* }\end{array}$ & $\begin{array}{l}\text { North displacement } \\
\text { \& difference from } \\
\text { MC* }\end{array}$ & $\begin{array}{l}\text { East displacement } \\
\text { \& difference from } \\
\text { MC* }\end{array}$ \\
\hline Minimum curvature & $\mathbf{6 4 4 7 . 4 7}$ & $\mathbf{0 . 0 0}$ & $\mathbf{8 6 7 8 . 9 8}$ & $\mathbf{0 . 0 0}$ & -7746.58 & $\mathbf{0 . 0 0}$ & $\mathbf{- 3 9 1 4 . 1 6}$ & $\mathbf{0 . 0 0}$ \\
\hline Tangential & $\mathbf{6 4 3 7 . 3 2}$ & $\mathbf{1 0 . 1 5}$ & $\mathbf{8 7 1 6 . 3 1}$ & $\mathbf{- 3 7 . 3 3}$ & $-\mathbf{7 7 6 8 . 6 2}$ & $\mathbf{2 2 . 0 4}$ & $\mathbf{- 3 9 3 0 . 7 2}$ & $\mathbf{1 6 . 5 6}$ \\
\hline Average angle & $\mathbf{6 4 4 7 . 6 0}$ & $\mathbf{- 0 . 1 3}$ & $\mathbf{8 6 8 8 . 6 0}$ & $\mathbf{- 9 . 6 2}$ & $-\mathbf{7 7 4 7 . 2 9}$ & $\mathbf{0 . 7 1}$ & $\mathbf{- 3 9 1 3 . 5 7}$ & $\mathbf{- 0 . 5 9}$ \\
\hline Balance & $\mathbf{6 4 4 7 . 0 7}$ & $\mathbf{0 . 4 0}$ & $\mathbf{8 6 8 8 . 1 1}$ & $\mathbf{- 9 . 1 3}$ & $-\mathbf{7 7 4 6 . 2 2}$ & $\mathbf{- 0 . 3 6}$ & $\mathbf{- 3 9 1 3 . 9 8}$ & $\mathbf{- 0 . 1 8}$ \\
\hline Radius of curvature & $\mathbf{6 4 4 7 . 5 2}$ & $\mathbf{0 . 0 5}$ & $\mathbf{8 6 8 8 . 0 3}$ & $\mathbf{- 9 . 0 5}$ & $-\mathbf{7 7 4 6 . 2 8}$ & $\mathbf{0 . 3 0}$ & $\mathbf{- 3 9 1 4 . 3 0}$ & $\mathbf{- 0 . 1 4}$ \\
\hline New model & $\mathbf{6 4 4 7 . 4 7}$ & $\mathbf{0 . 0 0}$ & $\mathbf{8 6 8 8 . 4 7}$ & $\mathbf{- 9 . 4 9}$ & $-\mathbf{7 7 4 7 . 0 2}$ & $\mathbf{0 . 4 4}$ & $\mathbf{- 3 9 1 3 . 6 8}$ & $\mathbf{- 0 . 4 8}$ \\
\hline
\end{tabular}

*Minimum Curvature Method 
International Advanced Research Journal in Science, Engineering and Technology

ISO 3297:2007 Certified

Vol. 3, Issue 11, November 2016

Table 4 Comparison of Accuracy of Various Calculation Method with New Model for Deep Kick off \& Build well (5I17)

\begin{tabular}{|c|c|c|c|c|c|c|c|c|}
\hline \multirow{2}{*}{$\begin{array}{l}\text { Method } \\
\text { Minimum } \\
\text { Curvature }\end{array}$} & \multicolumn{2}{|c|}{$\begin{array}{l}\text { TVD \& difference } \\
\text { from } M C^{*}\end{array}$} & \multicolumn{2}{|c|}{$\begin{array}{l}\text { HD \& difference } \\
\text { from } \mathrm{MC} *\end{array}$} & \multicolumn{2}{|c|}{$\begin{array}{l}\text { North displacement \& } \\
\text { difference from MC* }\end{array}$} & \multicolumn{2}{|c|}{$\begin{array}{l}\text { East displacement \& } \\
\text { difference from } \mathbf{M C} *\end{array}$} \\
\hline & 7224.75 & 0.00 & 2464.50 & 0.00 & 2445.65 & 0.00 & 287.29 & 0.00 \\
\hline Tangential & 7208.14 & 16.61 & 2531.56 & -67.03 & 2510.29 & -64.64 & 304.41 & -17.12 \\
\hline Average angle & 7224.86 & -0.11 & 2464.56 & -0.06 & 2430.65 & 15.00 & 278.91 & 8.38 \\
\hline Balance & 7224.50 & 0.25 & 2464.38 & 0.15 & 2439.50 & 6.15 & 281.27 & 6.02 \\
\hline $\begin{array}{l}\text { Radius of } \\
\text { curvature }\end{array}$ & 7224.52 & 0.23 & 2464.45 & 0.05 & 2440.28 & 5.37 & 283.12 & 4.17 \\
\hline New model & 7224.77 & -0.02 & 2464.49 & 0.01 & 2438.33 & 7.32 & 281.32 & 5.97 \\
\hline
\end{tabular}

* Minimum Curvature Method

\section{REFERENCES}

1) Rabia, H. (1987), "Well Engineering and Construction". Penn Well Pub. Co., Tulsa, Oklahoma, pp (433-511).

2) Brown,J. (2000), "Drilling Engineering ". Institute of Petroleum Engineering, Heriot-Watt University.1995, USA, pp (443-491).

3) Bourgoyne Jr, et al. (1986), "Applied Drilling Engineering". Society of Petroleum Engineers, USA, pp (351-472).

4) Baker Hughes INTEQ, (2007). "Drilling Engineering Workbook". A Distributed Learning Course 80270 H Rev. B, USA, pp (181284).

5) R.R. IIyasov, et al. (2014) "Automation of Optimal Well Trajectory Calculations” SPE, Moscow, Russia, 14-16 October

6) Carden, R. S. and Grace, R. D., (1999)," Horizontal and Directional Drilling PetroSkills", LLC. And OGCI Co. Tulsa, Oklahoma, USA, pp (1-113).

7) Jim Short, J. A. (1993),"Introduction to Directional and Horizontal Drilling". Penn Well Pub. Co., Tulsa, Oklahoma, pp (1-122).

8) Mitchell, B., (1995), "Advanced Oil Well Drilling Engineering". Handbook and Computer Programs. $10^{\text {th }}$ Edn., $1^{\text {st }}$ Revision - July. Mitchell Engineering Pub., USA, pp (292-463).

9) Siavoshi, J., (2004), "Drilling Technology". Memorial University of Newfoundland, Earth Sci. Dept., Canada, pp (94-105).

10) Gabolde, G. and Nguyen, J. P., (1999), "Drilling Data Handbook" $.7^{\text {th }}$ Edn., Institut Francais du Petrole Publ., Editions Technip, Paris, $\mathrm{pp}(413-455)$. 